\title{
Hydromorphone in the Management of Cancer-Related Pain: An Update on Routes of Administration and Dosage Forms
}

\author{
Maansi G. Kumar and Senshang Lin \\ College of Pharmacy and Allied Health Professions, St. John’s University, Jamaica, New York
}

Received July 21, 2007; Revised September 25, 2007; Accepted September 29, 2007; Published September 29, 2007.

\begin{abstract}
Pain is experienced by a majority of cancer patients. As life expectancy has increased in developed and developing countries, cancer-related pain has become a major health concern. Despite the use of the three-step analgesic ladder proposed by the World Health Organization, pain still remains under treated. Morphine, the gold standard against which all other opioids has been compared is considered the first choice for management of cancer-related pain. However, recently focus has shifted to the use of hydromorphone, a semi-synthetic derivative of morphine, which is more potent, more soluble and has a comparable side-effect profile. This review focuses on the use of hydromorphone for the management of cancer-related pain emphasizing on the various routes of administration as well as dosage forms, and providing a direction for the preference of a particular route depending on the need for a rapid effect and the individual's situation. Various approaches used to modify the release of hydromorphone from the drug delivery systems with the perspective of improving patient compliance are also being discussed.
\end{abstract}

\section{PAIN}

Pain is a complex experience (1) and one of the most feared symptoms associated with cancer $(2,3)$. It impacts the mobility, independence, psychological state and quality of life of the patient (3). The International Association for the Study of Pain defines pain as "an unpleasant sensory and emotional experience arising from actual or potential tissue damage or described in terms of such damage." It is alarming but true that more than 3.5 million cancer patients per day worldwide have significant cancer-related pain (4). This pain may arise from the cancer itself (extension into soft tissue, bone involvement, nerve injury), indirectly related to the cancer (muscle spasm, lymphedema), due to anticancer treatment (chronic post-surgical scar pain, chemotherapy-induced mucositis), or caused by a concurrent disorder (osteoarthritis) (5).

The World Health Organization has played a pivotal role in defining and disseminating the guidelines for the management of cancer-related pain. The three-step analgesic ladder (Figure 1) has had a major impact on the way cancer pain management is being organized and delivered $(3,5,6)$. It has been reported that about $60-90 \%$ of patients with cancer require an opioid analgesic to control their pain $(4,7)$. Despite the prevalence of "opiophobia" that is the exaggerated concerns about the risks of abuse and diversion, fear of side-effects, and inappropriate attitudes among physicians, patients and the family members of cancer patients $(4,8-10)$; opioids have been prescribed and are shown to be effective and safe in a majority of cancer patients (10).

Morphine is considered the gold standard for the management of pain because of its global availability, extensive clinical experience and ample pharmacokinetic and pharmacodynamic data (11). When used appropriately, about $80 \%$ of patients achieve adequate pain relief. This means that one in five people still suffer from pain and require an alternative opioid $(3,12)$. Hydromorphone has proved to be a suitable alternative for patients who cannot tolerate the side-effects of morphine or suffer from renal failure and asthma $(1,5,11,13-17)$.

Corresponding Author: Senshang Lin, Ph.D. College of Pharmacy and Allied Health Professions, St. John's University, 8000 Utopia Parkway, Jamaica; New York; USA, Tel: (718) 990-5344; E-mail: linse@,stjohns.edu 


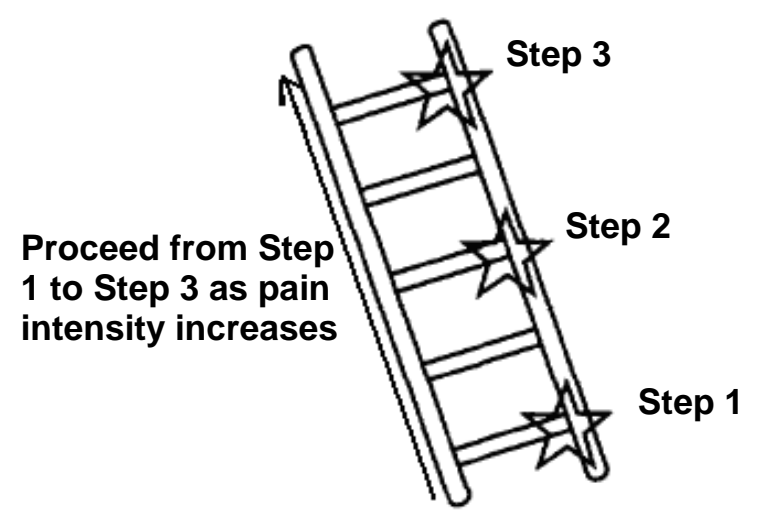

Figure 1. The three-step analgesic ladder proposed by World Health Organization for the management of pain. Step 1: Use of non-opioids like acetaminophen for mild pain; Step 2: Addition of weak opioids such as codeine, oxycodone or propoxyphen to the drug regimen in cases of moderate pain; Step 3: Use of strong opioids such as morphine, fentanyl or hydromorphone, along with the existing drug regimen for severe pain (adapted from reference 3)

\section{HYDROMORPHONE}

Hydromorphone is a semi-synthetic derivative of morphine $(1,13,18-21)$ that has been in clinical practice since the 1920 s $(22,23)$. Structurally, in hydromorphone the $\mathrm{C}_{6}$ hydroxyl group of morphine has been substituted with a carbonyl group and the double bond at the $\mathrm{C}_{7-8}$ position has been removed (Figure 2), yielding an antinociceptive potency that far exceeds that of the original compound $(13,24)$.

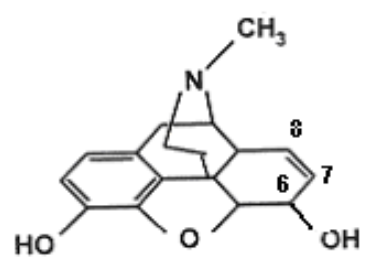

A

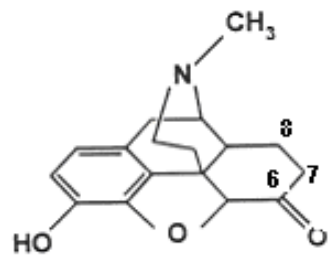

B
Figure 2. Structures of (A) morphine and (B) hydromorphone

The mechanism of action, chemistry, pharmacology, pharmacokinetics, pharmacodynamics and side-effects of hydromorphone has been described by various researchers in detail $(5,11,25-28)$. Clinical studies have reported that hydromorphone is approximately five to seven times more potent than morphine, with a shorter duration of analgesia and a comparable side-effect profile $(5,19,21,29)$. Numerous studies have also been conducted comparing hydromorphone to other opioids when administered by the oral $(30,31)$, intravenous (32-35), intramuscular (36), subcutaneous (22), and epidural (37-40) routes. In the United States, hydromorphone is available commercially in various dosage forms (Table 1) (28).

Oral dosage forms of hydromorphone are available in the form of solution $(1 \mathrm{mg} / \mathrm{mL})$ and immediate-release tablets $(2,4$ and $8 \mathrm{mg})$. Parenteral ampoules (1, 2 and $4 \mathrm{mg} / \mathrm{mL}$ ) and suppositories $(3 \mathrm{mg})$ are also available. A concentrated injection containing $10 \mathrm{mg} / \mathrm{mL}$ of hydromorphone hydrochloride is preferred when a large dose of morphine equivalent has to be administered parenterally $(1,29)$ or when the volume of the opioid solution to be injected must be limited (41). In 2004, the Food and Drug Administration approved of a controlled-release formulation of hydromorphone (Palladone ${ }^{\mathrm{TM}}$ ) for once-daily administration. However, recently this dosage form has been withdrawn from the market because of concerns of the controlled-release mechanism being harmed when consumed with alcohol leading to dose-dumping and potential adverse effects (42-45). The usual adult oral dosage of hydromorphone is $2 \mathrm{mg}$ every 4-6 hours as necessary and doses of up to $4 \mathrm{mg}$ or more may be administered every 3-6 hours as indicated and tolerated. By subcutaneous injection, $2 \mathrm{mg}$ of hydromorphone is administered every 4-6 hours as necessary and for severe pain. Rectally, the usual dose is $3 \mathrm{mg}$ administered in the form of a suppository every 6-8 hours as necessary (28). However, for the treatment of cancer-related pain there is no standard dose of hydromorphone. The right dose is that which brings relief to the patient because pain intensity and response varies between patients and can not be generalized (5).

Years of research by investigators in cancer pain management has lead to the conclusion that most cancer pain patients benefit from and often require an alternate route of administration during the progression of the disease. Routes of administration and different dosage forms are often 
required for convenience and for better control of pain intensity and adverse effects $(5,28,46,47)$. To address this, the various routes of administration of hydromorphone and an update on their dosage forms will be reviewed.

\section{ROUTES OF ADMINISTRATION FOR HYDROMORPHONE}

\section{Oral route}

The oral route is the simplest of all drug administration routes and is the preferred route for the administration of hydromorphone $(1,2,5,28,48$ 51). The obvious advantages of the oral route are safety, convenience, low cost, high patient compliance and patient independence $(1,48,52)$. Orally, hydromorphone from aqueous solution is absorbed by first-order kinetics mainly from the upper small intestine. A low bioavailability of $62 \pm$ $33 \%$ due to extensive first-pass metabolism has been reported $(5,25,28,53,54)$. Immediate-release tablets of hydromorphone are known to show their effect within 30 minutes of administration $(5,26,28,55)$ and the effect lasts for about 4 hours.

Thus, to overcome the need for repetitive dosing with the immediate-release tablets (55-58), a modified-release product of hydromorphone, namely Palladone ${ }^{\mathrm{TM}}$ was introduced $(11,59)$.

Palladone ${ }^{\mathrm{TM}}$ is a once a day formulation of hydromorphone that can be administered by sprinkling it on food or through a feeding tube, if necessary. These capsules contain many individual pellets which are formulated by a controlled-release melt extrusion technology and contain hydromorphone hydrochloride as the active, and a methacrylate copolymer, ethylcellulose and stearyl alcohol as the excipients to regulate the dissolution as well as the absorption of hydromorphone over a 24-hour period. These capsules are filled with the identical pellets using different fill weights to achieve different strengths of 12, 16, 24 and $32 \mathrm{mg}$. After ingestion, the capsule dissolves in the gastrointestinal tract within 5 minutes to release some hydromorphone from the surface of the pellet, which provides immediate absorption and pain relief. As the pellets move down the gastrointestinal tract, fluid penetrates the matrix and dissolves the hydromorphone. Once dissolved, hydromorphone begins to diffuse from the matrix and is available for systemic absorption. The absorption of hydromorphone from this controlled-release formulation is characterized by a biphasic profile, i.e., a relatively rapid rise to an initial peak concentration followed by a second broader peak with therapeutic plasma concentrations being maintained for up to 24 hours (Figure 3). This biphasic absorption profile was observed to be significantly different from that of an immediaterelease tablet (Figure 3). Moreover, the pharmacokinetic profile of hydromorphone following the administration of Palladone ${ }^{\mathrm{TM}}$ was found to be proportional to the dose administered.

In a study comparing $12 \mathrm{mg}$ Palladone ${ }^{\mathrm{TM}}$ capsules dosed every 24 hours to $3 \mathrm{mg}$ immediate-release hydromorphone tablets dosed every 6 hours, it was found that the two treatments were equivalent in terms of extent of absorption with area under the curve values of 34.9 and $34.4 \mathrm{ng} \mathrm{h} / \mathrm{mL}$, respectively (59). The controlled-release characteristics of Palladone ${ }^{\mathrm{TM}}$ capsules also resulted in lower steadystate peak levels, higher trough levels, and an approximately two-three folds reduction in the fluctuation when compared to immediate-release hydromorphone tablets (Figure 3) (11,43,58-60). However, due to safety concerns of dose-dumping during alcohol consumption, this controlled-release dosage form has been withdrawn from the market and the company has implemented a plan to reformulate the product (43).

Another controlled-release product for once-daily dosing of hydromorphone but using osmotic technology is being developed $(43,61)$. This system consists of a semi-permeable membrane surrounding a bilayer tablet core; one layer containing hydromorphone, and the other an osmotically active component. A small hole is drilled through the membrane on the side adjacent to the drug layer (Figure 4).

Once ingested, water diffuses across the membrane, and a gel-like suspension is formed in the drug layer. As the osmotic layer expands, hydromorphone is pushed through the orifice at a near constant rate into the lumen of the gastrointestinal tract for absorption. The rate at which hydromorphone is released equals that at which osmotic pressure results from water entering the tablet core and is independent of gastric motility or $\mathrm{pH}$. Since the osmotic gradient remains the same, the release rate of hydromorphone is constant. After release of hydromorphone, the non-absorbable tablet shell is then passed in the stool. 
Table 1. Various dosage forms of hydromorphone available commercially in the United States

\begin{tabular}{lllll} 
Route & Dosage form & Brand name & Dosage or concentration & Comments \\
\hline Oral & Solution & Dilaudid & $5 \mathrm{mg} / 5 \mathrm{~mL}$ & \\
& $\begin{array}{l}\text { Immediate- } \\
\text { release tablet }\end{array}$ & Dilaudid & 2,4 and $8 \mathrm{mg}$ & \\
$\begin{array}{l}\text { Controlled- } \\
\text { release capsule }\end{array}$ & Palladone & $12,16,24$ and $32 \mathrm{mg}$ & $\begin{array}{l}\text { withdrawn in 2005 } \\
\text { (drug-alcohol } \\
\text { interaction) }\end{array}$ \\
& $\begin{array}{l}\text { Powder for } \\
\text { reconstitution }\end{array}$ & Dilaudid-HP & $250 \mathrm{mg}$ & \\
Ampoule & Dilaudid & $1,2 \mathrm{and} 4 \mathrm{mg} / \mathrm{mL}$ & for opioid-tolerant \\
Rectal & Suppository & Dilaudid & $3 \mathrm{mg}$ & patients \\
\hline
\end{tabular}

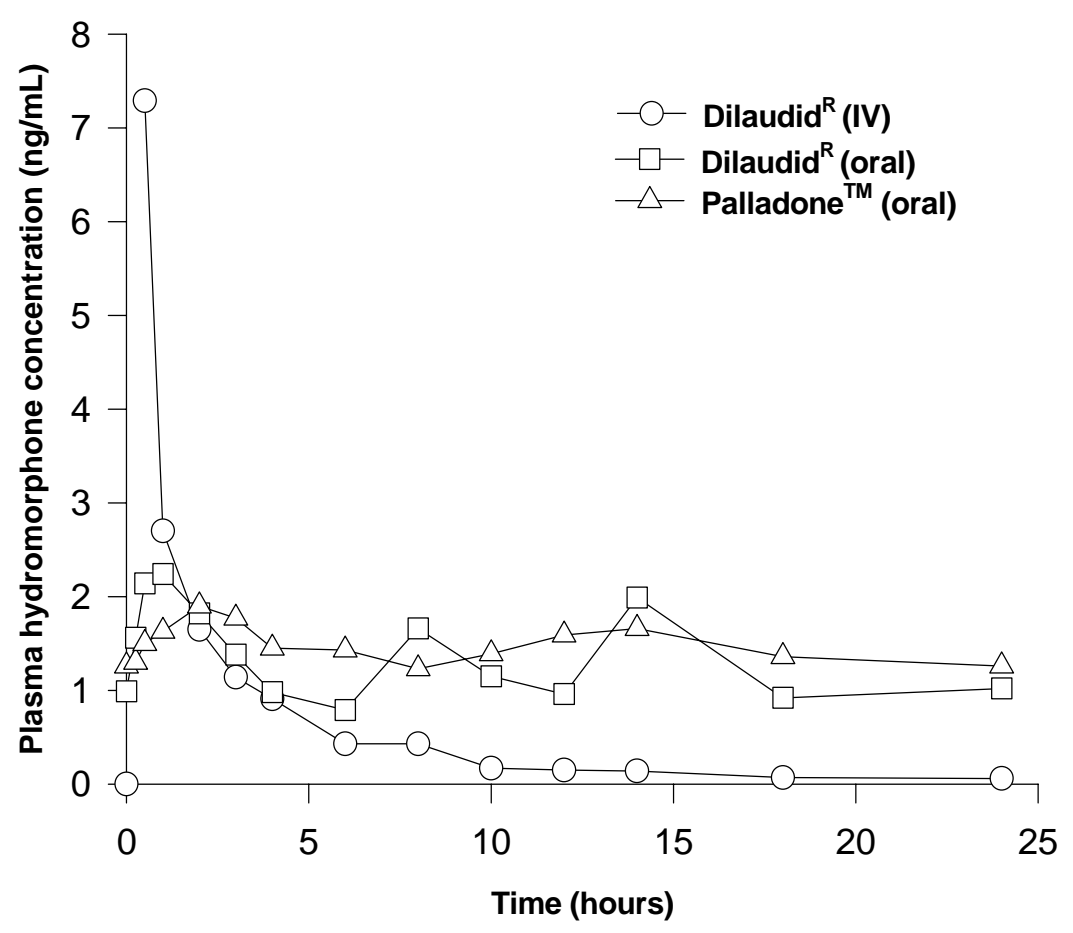

Figure 3. Mean plasma hydromorphone concentrations versus time profiles after a single intravenous administration of hydromorphone hydrochloride $\left(\right.$ Dilaudid $^{\mathrm{R}}$ ), $12 \mathrm{mg}$ controlled-release capsule of hydromorphone hydrochloride administered once a day (Palladone ${ }^{\mathrm{TM}}$ ) and $3 \mathrm{mg}$ immediate-release tablet of hydromorphone hydrochloride $\left(\right.$ Dilaudid $^{\mathrm{R}}$ ) dosed every six hours (adapted from references 11,59) 


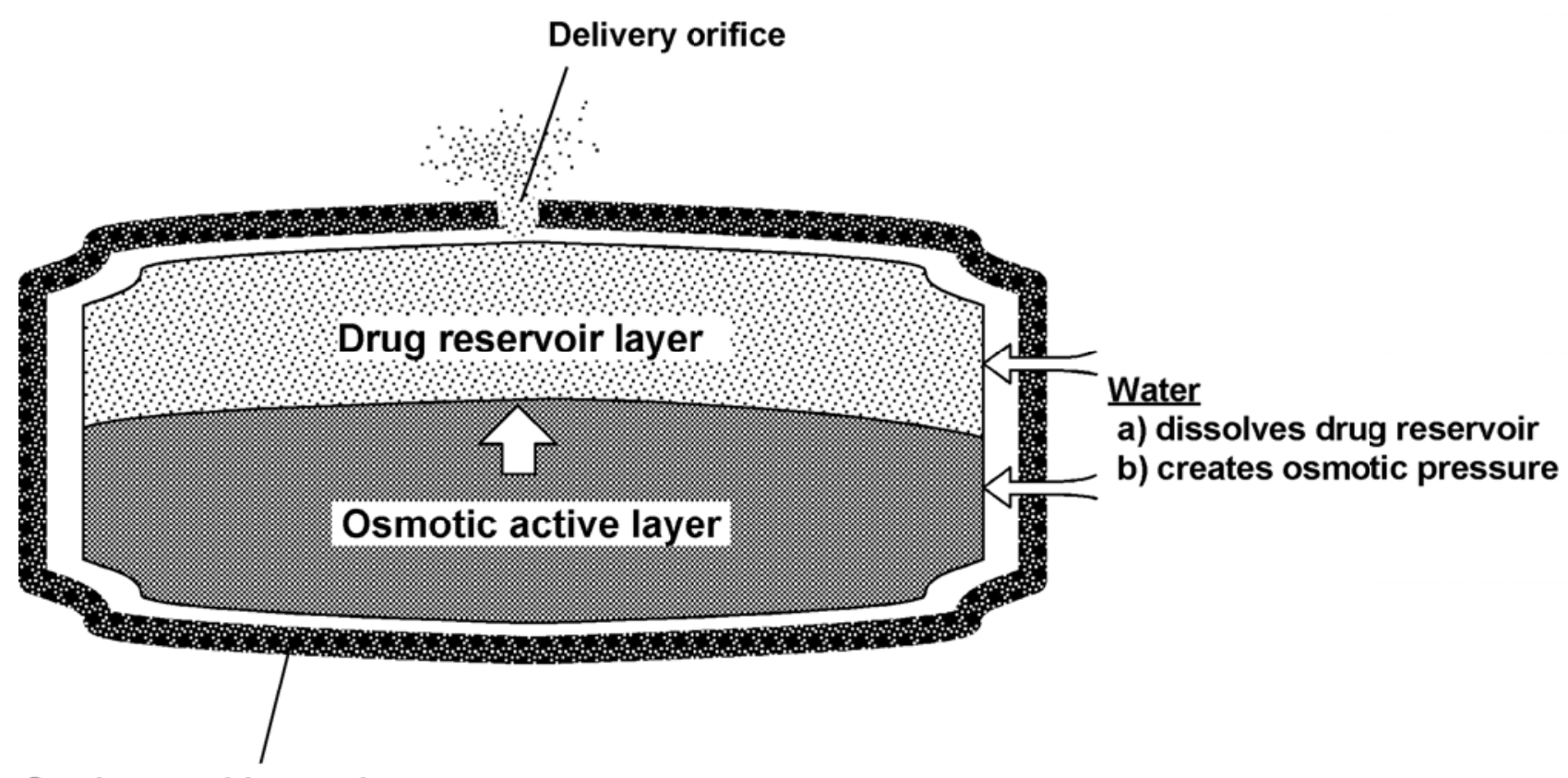

\section{Semipermeable membrane}

Figure 4. Schematic of the $\operatorname{OROS}^{\circledR}$ controlled-release hydromorphone formulation tablet

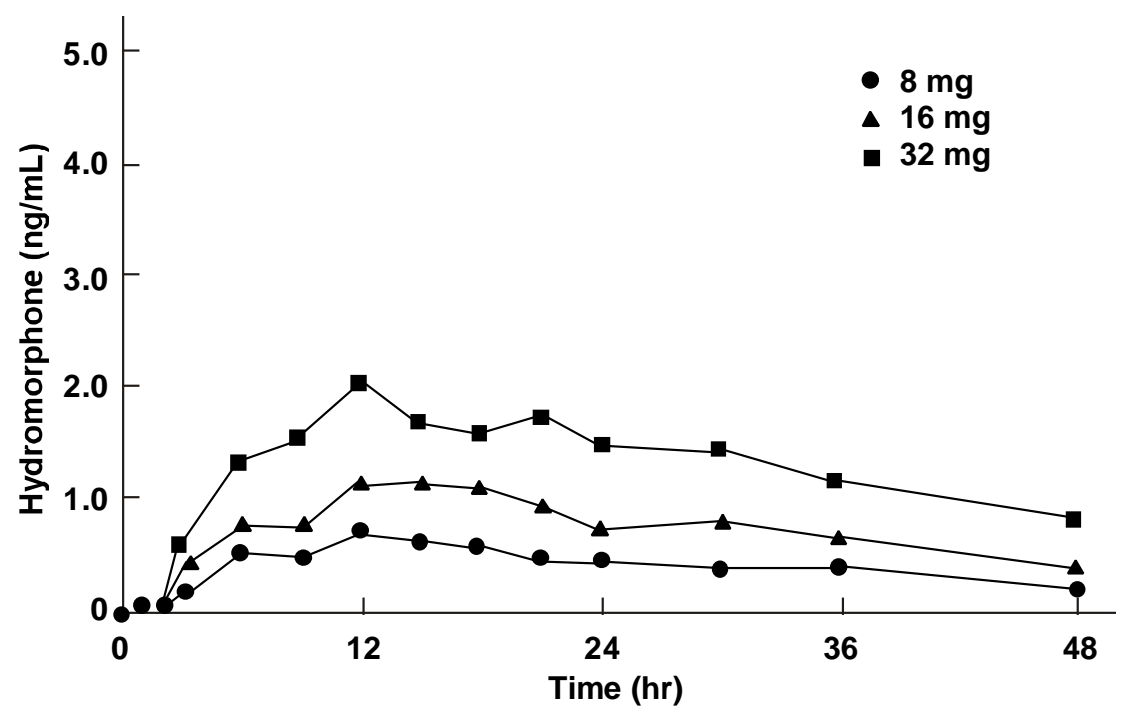

Figure 5. Mean plasma hydromorphone concentrations of single doses of 8,16 , and $32 \mathrm{mg} \operatorname{OROS}^{\circledR}$ formulations of hydromorphone (adapted from reference 63) 
The pharmacokinetics of this system has been characterized by Drover and his colleagues $(58,62)$ and a dose-dependent analgesic response beginning at 6 hours and lasting for more than 24 hours has been documented (Figure 5) (55).

Both these modified-release products have a half-life of one hour and take three-four folds more time than immediate-release hydromorphone tablets to reach peak plasma levels $(55,56,64)$. Since modified-release products provide sustained hydromorphone concentrations in blood for prolonged periods of time, in comparison to immediate-release hydromorphone preparations, patients on modified-release products need not be disturbed at night to take medication in order to maintain effective analgesic levels. In addition, patient compliance to such dosage forms is much higher, because less number of dosages needs to be consumed by the patient. The most important advantage of these formulations is that the chances of breakthrough pain are reduced. Modified-release products have thus enhanced the attractiveness of the oral route and improved the overall quality of life of patients. $(43,50,51,56,58,59)$. However, it has been reported that about $33-70 \%$ of cancer patients require alternatives to the oral route of administration (54) especially during cases of severe dysphagia, intractable vomiting, complete gastric stasis, bowel obstruction or when malabsorption syndrome is present $(2,5,28,29,49,52)$.

\section{Parenteral route}

Parenteral administration of hydromorphone is the most frequent alternative to oral therapy. This route is indicated in patients with difficulty in swallowing, nausea, vomiting, bowel obstruction and altered consciousness $(1,2,4,28)$. Hydromorphone can be administered by the intravenous, intramuscular and subcutaneous routes with an oral to parenteral equianalgesic ratio of 5:1 (25). After intravenous injection of hydromorphone, peak plasma levels occur soon after administration, but these levels decline rapidly owing to the fast distribution into liver, spleen, kidney and skeletal muscles $(28,52)$. The onset of analgesia occurs within 5 minutes and the maximum analgesic effect occurs 8 to 20 minutes after maximum plasma concentration $(19,25,28,32)$. A log-linear dose-dependent relationship is observed (19) and a three exponential equation is commonly used to describe the disposition of intravenous hydromorphone in humans (65). However, when administered intramuscularly, the absorption of hydromorphone is erratic and does not offer any pharmacokinetic and/or analgesic advantage to the patient (5).

In addition, the intramuscular route is not advised for emaciated, cachectic, or elderly patients since they already have reduced muscle mass (28). On the other hand, the subcutaneous route is reported to be a safe and effective alternative (66). An added advantage of the subcutaneous route is that since hydromorphone has good water solubility, it can be administered in higher daily doses in very low volumes $(48,49)$. The subcutaneous administration of hydromorphone has been found to have $78 \%$ of the bioavailability of intravenous dosing $(11,28)$.

Due to the short duration of action of hydromorphone, injections by all three parenteral routes would need to be repeated regularly, thereby becoming painful for the patient, time consuming for the caregivers, and difficult to maintain in the home setting (48). So, to provide for stable blood levels of the drug, continuous infusions of hydromorphone have been administered both subcutaneously and intravenously $(7,67,68)$. No differences have been found in terms of the estimates of pain intensity, pain relief, mood, drug levels, extent of sedation and the number of injections required for breakthrough pain between the two methods of continuous infusion administration (68). However, since the intravenous route is complicated to maintain and requires prolonged venous access and close supervision, subcutaneous infusions are generally preferred $(49,69)$. They appear to carry all the advantages of continuous intravenous infusion with the added benefits of greater mobility, management on an outpatient basis, as well as less pain, risk and expense $(2,5,29,66,68)$.

Although parenteral infusions do provide for stable drug levels, the parenteral administrations are usually cumbersome, expensive and dependent upon the availability of vascular or subcutaneous catheters, infusion pumps, and trained nursing and pharmacy personnel (4). These routes are also contraindicated in patients with renal or metabolic abnormalities, and can not provide pain control in patients with nerve damage, incidental pain, or pain due to psychological distress (2). As a result, if implantable controlled-release systems were 
introduced to deliver hydromorphone then it would be possible to eliminate hardware, personnel as well as expense associated with infusion administration. This approach of controlled-release using polymeric systems eliminates the need for daily clinical visits, reduces the costs and time constraints of treatment, increases patient satisfaction, reduces side-effects, improves quality of life and reduces the risk of illicit diversion $(70,71)$.

Various researchers have used polymeric devices to deliver hydromorphone at a constant rate. Lesser and his colleagues (4) employed a low temperature solvent casting technique to formulate ethylene vinyl acetate copolymer disks containing $50 \%$ hydromorphone by weight. They studied the release characteristics of the disks of different heights and diameters, either uncoated or coated with poly (methyl-methacrylate) and with or without a central uncoated channel. By fabricating a cylindrical opening along the axis of the cylindrical matrix they created an internal wall which had no coating, and were able to achieve near constant delivery of hydromorphone. A similar stable plasma hydromorphone concentration which was sustained for four weeks following the subcutaneous implantation of the system was observed in rabbits (Figure 6) (4,70,72).

Additionally, Sendil et al. (73) used the copolymer poly L-lactide-co-glycolide (PLGA) to fabricate an implantable rod type delivery system containing hydromorphone. A zero-order release from the rods that contained PLGA in the ratio of
85:15 was observed. When implanted intrathecally in rats, this system produced a rapid onset of analgesia and prolonged antinociception for up to five days (73). However, the introduction of polymeric systems requires minor surgical procedure and are designed to meet only the stable dosing needs of patients in pain $(4,70)$. To address the problem of breakthrough pain, Enting et al. employed an injection pen similar to the one widely used for insulin administration (74). The pen allows for easy and accurate subcutaneous drug delivery, with the smallest injectable volume of $50 \mu \mathrm{L}$ to be equivalent to one click. For larger volumes, the dosage knob can be turned clockwise until the desired number of clicks appears. The total amount of drug is then injected through a very thin needle of 25 gauge or larger by pressing the dosage knob once. The advantage of this system includes the ability to administer higher doses of hydromorphone at a moderate cost (74).

Furthermore, the parenteral route does have its limitations in terms of causing discomfort to patients, and in some cases can result in infections or tissue irritation. Moreover, the resource costs of this method of administration are quite high as compared to oral administration (52), and this route can not be used in patients with generalized edema, coagulation disorders, severe immuno-suppression, poor peripheral circulation or those who develop erythema, soreness and sterile abscesses $(3,48,49,75)$.
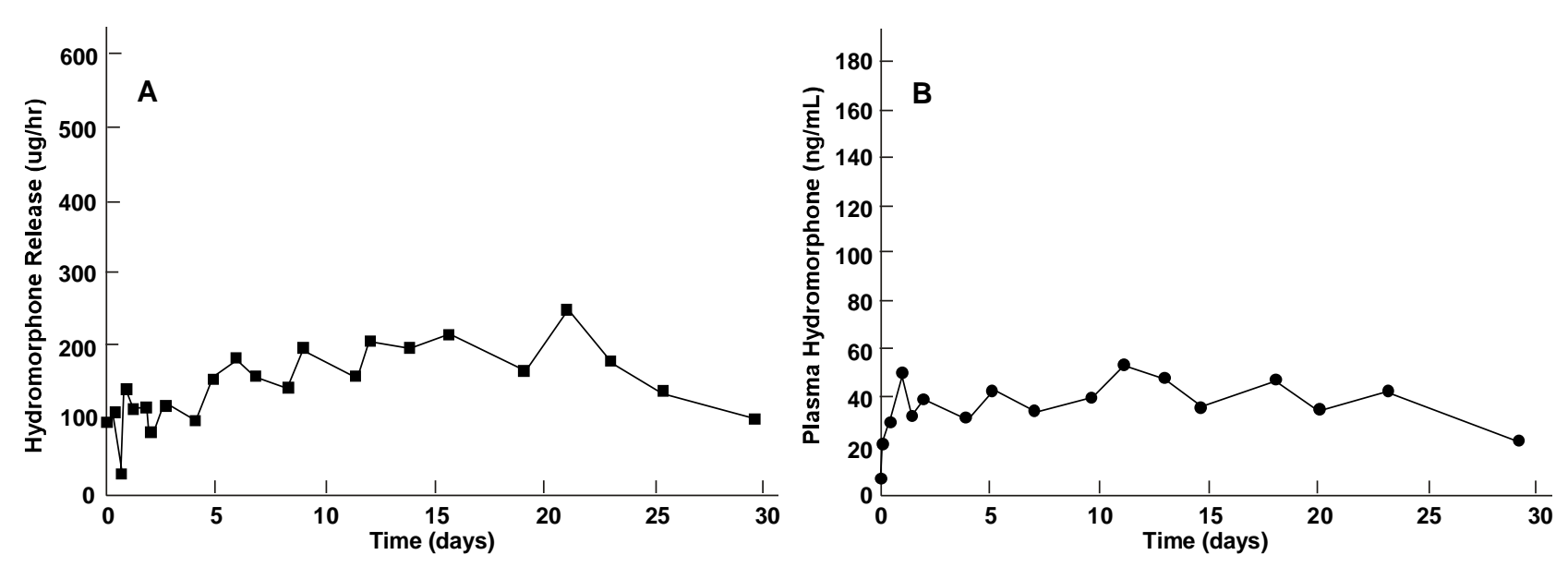

Figure 6. (A) In-vitro release of hydromorphone from coated polymer disk with a central uncoated channel and (B) plasma hydromorphone concentrations in rabbits after subcutaneous implantation of the polymer disk (adapted from reference 4) 


\section{Rectal route}

The rectal route of administration can be considered an alternative for patients when the oral route is contraindicated $(29,49)$, or when the fear of needles limits the use of the parenteral route. Potentially rectal administration has the pharmacokinetic advantage of bypassing first-pass hepatic metabolism (49).

Ritschel and his colleagues have determined the absolute bioavailability of rectal administration of hydromorphone suppository in humans. They reported a maximum concentration of $3.53 \pm 1.36 \mathrm{ng} / \mathrm{mL}$, with the time taken to reach maximum concentration as $1.41 \pm 0.79$ hours, a bioavailability of $36 \pm 0.29$ percent and a half-life of 3.8 hours (Figure 7). The low bioavailability and large inter-individual variation observed when hydromorphone is administered rectally is due to various factors, namely the type of preparation, the $\mathrm{pH}$ of the solutions used, the presence of feces in the ampulla, the condition of the mucosa, the placement of the agent, and the concurrent use of lubricants $(5,28,49,53)$.

Although the rectal route is as effective as the oral route, mild mucosal hyperemia and conditions like diarrhea, colostomy, hemorrhoids and anal fissures may limit the use of this route of administration $(5,28,48,49,53)$.

\section{Transdermal route}

The stratum corneum of the skin is an excellent barrier to transdermal transport of solutes (76), but it offers an exciting possibility of achieving prolonged, stable blood levels of the drug in a noninvasive manner $(48,52)$. This route is much more convenient for patients as compared to the parenteral, rectal or spinal routes, and can also be used in patients who are unable to tolerate oral administration or who are too sick and confused to be taught how to use an infusion pump (52).

Ideally, for administration by this route, the drug should have a favorable oil/water partition coefficient, low molecular weight and sufficient potency $(5,76)$. Since hydromorphone does not possess all the required attributes for successful transdermal delivery, it remains undetected during the first few hours but later steady-state concentrations are achieved and maintained for 24 hours without any depot formation in the skin. An absolute bioavailability of $86 \%$ by the transdermal route has also been reported in rabbits (26). To achieve rapid therapeutic drug levels transdermally physical and chemical enhancement techniques are generally employed.

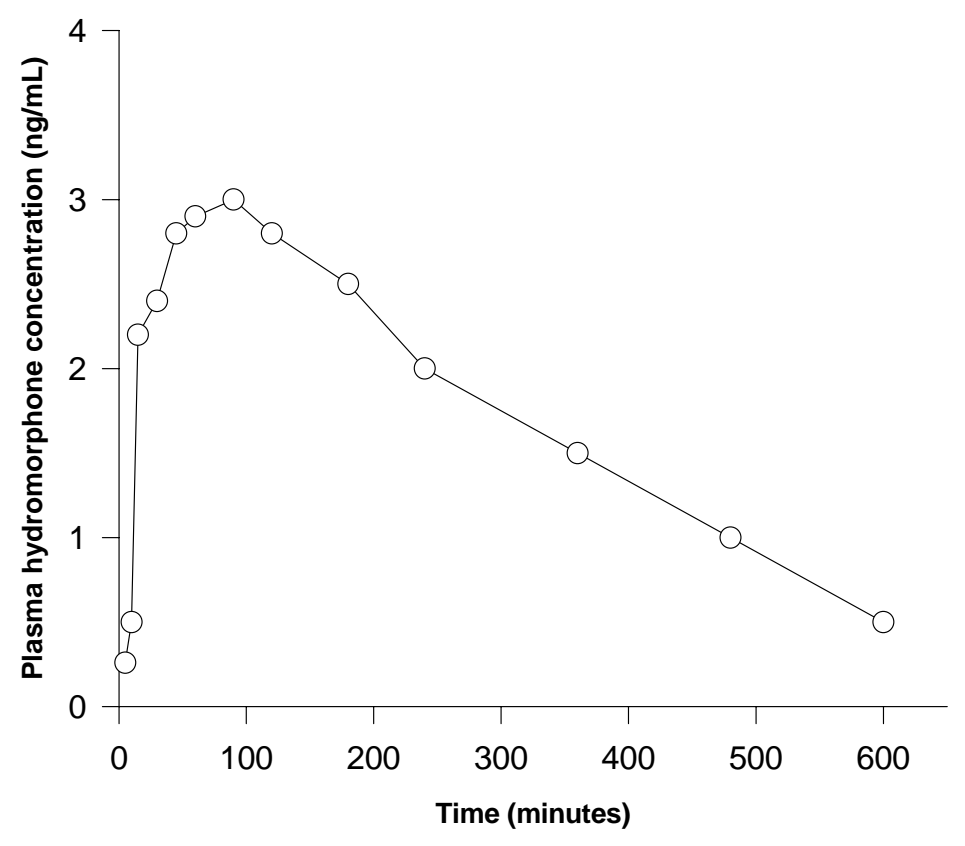

Figure 7. Mean plasma hydromorphone concentrations after rectal administration of $3 \mathrm{mg}$ suppository in humans (adapted from reference 53) 
The physical enhancement techniques include iontophoresis, sonophoresis, use of microneedles and mechanical abrasion while the chemical enhancement techniques include the use of permeation enhancers. Padmanabhan et al. have used the technique of iontophoresis, i.e., use of an external electric field to propel charged molecules through the skin, to deliver hydromorphone from an aqueous solution and hydrogel formulation (77). Also, Smith and his colleagues have used the PassPort ${ }^{\mathrm{TM}}$ technology, i.e., the thermal ablation of the stratum corneum to create aqueous micropores for the passive permeation of hydromorphone. By this method, the apparent rate of delivery of hydromorphone was found to be dependent on the area of microporated skin and micropore density. The mean pharmacokinetic curve for the $1 \mathrm{~cm}^{2}$ patch $\left(120\right.$ micropores $\left./ \mathrm{cm}^{2}\right)$ demonstrated a rapid rise in serum hydromorphone levels after the patch was applied, reaching a mean steady-state level of $3.1 \mathrm{ng} / \mathrm{mL}$ after 8 hours. This level was maintained until patch removal at 24 hours, after which the hydromorphone serum half-life was measured as 3 hours. The elimination rate was comparable to that found after intravenous infusion of hydromorphone, indicating little or no depot of hydromorphone in the skin. Analysis of the residual drug in the patch showed that an average of $82 \%$ of hydromorphone was delivered from the patch into the skin over the 24-hour period (78).

Cutaneous reactions to the transdermal patch like erythema and itching, an unstable pain condition, and generalized edema in a patient are conditions that may limit the use of the transdermal route $(48,49)$. Also, additional costs may be involved for the disposal of the patches because of fear of improper use of the residual drug in the patch (52).

\section{Intranasal route}

The advantages of administering drugs by the intranasal route are obvious and include ease of administration, rapid onset, avoidance of degradation in the gastrointestinal tract and better patient control $(47,54)$.

Hydromorphone, as an aqueous solution buffered to $\mathrm{pH} 4.0$ with $0.2 \%$ sodium citrate and $0.2 \%$ citric acid, has been delivered as a metered dose spray. Hydromorphone was observed to be well-tolerated and rapidly absorbed after intranasal administration. Plasma concentrations of $2.8 \pm 0.7$ and $5.3 \pm 2.3 \mathrm{ng} / \mathrm{mL}$ have been reported after the administration of 1 and $2 \mathrm{mg}$, respectively, of hydromorphone every 6 hours. The median time to peak concentration was observed to be 20 minutes for multiple doses and also dose proportionality was observed for the 1 and $2 \mathrm{mg}$ doses (Figure 8). Absolute bioavailabilities of $52 \%$ and $57 \%$ after single intranasal doses of 1 and $2 \mathrm{mg}$ have been reported, respectively. Allergic rhinitis was however found to affect pain management strategies for intranasal hydromorphone and this should be kept in mind when administering hydromorphone through the intranasal route $(47,54,79)$.

\section{Intraspinal/Intrathecal route}

Intraspinal delivery of opioids is a proven method of pain management for severe cancer-related pain with morphine being the only drug approved by the Food and Drug Administration (21,80-83). This route enables to target specific sites of action where nociceptive signals are encoded in the spinal cord $(73,84)$. Administration through this route minimizes the central side-effects such as sedation, nausea, and severe constipation. Furthermore, this route is employed when unmanageable side-effects with increasing doses of oral or parenteral opioids, or little or no pain relief with increasing dosage of opioids is an issue $(5,73,80,81,85)$. As spinal administration requires special expertise and equipment for catheter placement, this route is practically used only for a minority of patients cared for in specialized settings. The risk of infections and other complications also results in employing this route only when all other less invasive options have been exhausted $(5,81)$.

Hydromorphone has the potential for being delivered by the intraspinal route as it has a greater potency and no active metabolites. These characteristics results in rapid entry of hydromorphone into the spinal cord, shorter onset of analgesia and fewer side-effects $(5,21)$. Hydromorphone delivered via this route has been detected in blood one minute after administration and its concentration in the cerebrospinal fluid is observed to peak after one hour of administration (28). Studies conducted conclude that hydromorphone is a safe and clinically useful analgesic for intraspinal delivery $(21,82)$. These observations have made investigators consider hydromorphone as a first-line agent for intraspinal delivery (83). 


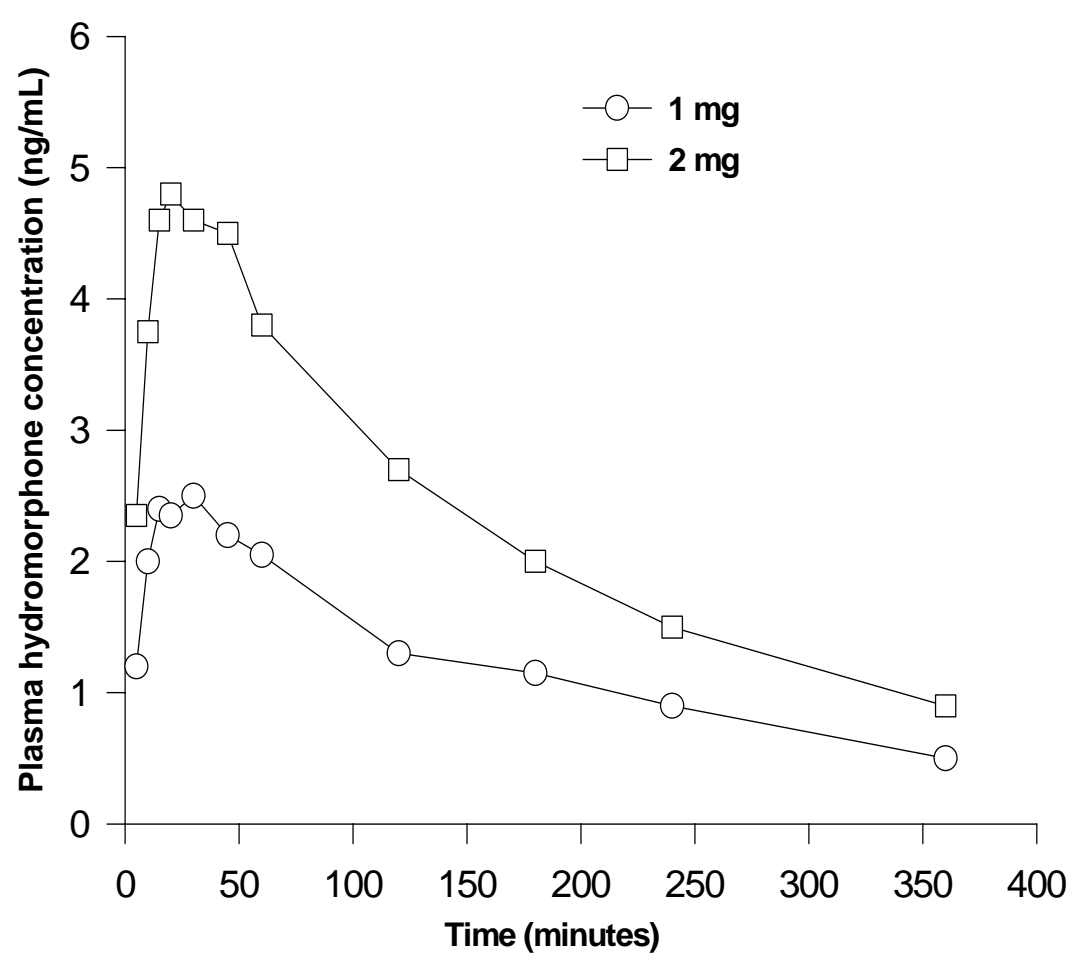

Figure 8. Mean plasma hydromorphone concentrations after intranasal delivery of hydromorphone hydrochloride in humans at dose levels of 1 and $2 \mathrm{mg}$ (adapted from reference 47)

\section{INAPPROPRIATE ROUTES OF ADMIN- ISTRATION FOR HYDROMORPHONE}

\section{Sublingual/Buccal route}

The sublingual and buccal routes are known to be potential routes for the delivery of drugs because they are highly vascular and avoid first-pass metabolism (49). However, absorption at this site is favored by the drug being poorly ionized at the $\mathrm{pH}$ of saliva, and possessing a high lipophilicity, neither of which applies to hydromorphone. Therefore, the sublingual and buccal routes are unlikely to be preferred routes of administration for hydromorphone (28).

\section{Inhalational route}

The inhalation route is non-invasive and associated with rapid absorption. However, the large variation in absorption observed due to nebulizer design, amount of drug swallowed, and the inspiratory/expiratory ratio of the respiratory cycle is a major disadvantage (76). Nebulized hydromorphone has been reported to have very low bioavailability and hence should not be used for pain control (28). Also, extremely ill patients, those in comatose states, or those suffering from asthma and feelings of claustrophobia caused by wearing a mask will not be able to use this route (49).

\section{COMPARISON BETWEEN THE FEASIBLE ROUTES OF ADMINISTRATION FOR HYDROMORPHONE}

Numerous studies have been conducted to compare hydromorphone to other opioids (22,30-40) however, very limited work has been done to compare the different available routes for the administration of hydromorphone in patients with cancer-related pain. A review of the available literature shows that when a comparison between the epidural and intramuscular routes was conducted, the epidural route was found to be superior in terms of duration of analgesia and need for rescue analgesia (86). Furthermore, when the subcutaneous and intravenous infusions routes were compared in a randomized, double-blind, doubledummy study in 20 patients, no difference between 
the groups in terms of analgesia, side-effects and rescue analgesia was observed (68). Another study comparing the oral and intramuscular routes for administration of hydromorphone in a randomized, double-blind, crossover study of 96 patients with chronic cancer pain showed that at equianalgesic doses more side-effects were observed with the intramuscular administration (23).

\section{TRENDS IN THE USE AND ABUSE OF HYDROMORPHONE}

It is most often thought that the abuse potential of opioid analgesics is such that increases in medical use of such drugs will inevitably lead to increases in their abuse. A retrospective survey by Joranson et al. of the medical records stored in the databases of the Drug Abuse Warning Network (DAWN; source of abuse data) and the Automation of Reports and Consolidated Orders System (ARCOS; source of medical use data) during the period of 1990 to 1996 showed a steady increase in the medical use of opioid analgesics in spite of a relatively low and stable level of abuse. Specifically, these authors reported a $15 \%$ decrease in the abuse of hydromorphone despite a $19 \%$ increase in its medical use during this time period. A similar trend of increase in the medical use with a decrease in abuse was also reported for other opioids such as morphine, fentanyl and oxycodone (8). Gilson et al. continued this study for the period of 1997 to 2002, but their study showed a different trend; an increase of $96 \%$ in hydromorphone medical use was accompanied with a $341 \%$ increase in its abuse during the specified time period (87). However, as compared to other abused drugs, opioid analgesics accounted for only $3.8 \%$ and $9.0 \%$ of the total DAWN mentions in 1996 and 2002, respectively $(8,87)$. It should be kept in mind that despite the potential of opioids to be abused, their use in cancer patients to relieve cancer-related pain must not be limited; rather the concern for abuse should be addressed by identifying the causes and sources of diversion, without interfering with legitimate medical practice and patient care.

\section{CONCLUSION}

Hydromorphone can be given through various routes of administration as it has the potential to be absorbed from almost all types of mucosa. However, the preference for a particular route of administration should depend on the condition of the patient, the rapidity with which relief is required, and the accessible routes of administration in the concerned patient. It can be concluded that although oral administration can manage cancer-related pain, alternative routes are generally required or preferred by most patients. For instance, when a rapid analgesic effect is an important clinical consideration, or when patients require very high doses of hydromorphone, or when contraindications to oral administration exist, then the parenteral routes should be selected. Also, when stable pain control can be achieved at a dose that can be administered by a non-invasive route, patients should be switched to such a route and discharged, so as to make home-management of cancer-related pain a possibility. Additionally, the intraspinal route should only be selected in cases of persistent doselimiting toxicity from systemic opioid therapy. Also, the sublingual, buccal or inhalation routes should be avoided when hydromorphone is to be administered. Furthermore, for the management of chronic cancer-related pain, implants and once-daily formulations of hydromorphone should be selected, as these products will eliminate the problems associated with the need for "around the clock" dosing. In other words, since hydromorphone implants and once-daily formulations will provide sustained hydromorphone levels, they will reduce the need for repetitive drug intake and hence improve the management of cancer-related pain. They will also allow for increased patient mobility as patients need not be attached to catheters to receive their dose. Moreover, the need for hardware, personnel, and expense associated with subcutaneous and intravenous pumps will be totally eliminated. Summarizing, it can be said that hydromorphone should no longer be considered as a second-line agent for management of cancer-related pain, but an equal to morphine, with various potential routes of administration and available dosage forms.

\section{REFRENCES}

[1]. Robert G. Twycross. Opioid analgesics in cancer pain: current practice and controversies. Cancer Surv, 7:29-53, 1988. 
[2]. Barbara C. Poniatowski. Continuous subcutaneous infusions for pain control. J Intraven Nurs, 14:30-35, 1991.

[3]. S Ahmedzai. New approaches to pain control in patients with cancer. Eur J Cancer, 33 Suppl 6:S8-14, 1997.

[4]. Glenn J. Lesser, Stuart A. Grossman, Kam W. Leong, Hungnan Lo, Susan Eller. In vitro and in vivo studies of subcutaneous hydromorphone implants designed for the treatment of cancer pain. Pain, 65:265-272, 1996.

[5]. Hasirci, V., Sendil, D., Goudas, L.C., Carr, D.B., Wise, D.L., Controlled Release Pain Management Systems, in Wise DL (ed), Handbook of Pharma- ceutical Controlled Release Technology. Marcel Dekker INC, New York, NY, pp 787-806, 2000.

[6]. M T. Smith. Neuroexcitatory effects of morphine and hydromorphone: evidence implicating the 3glucuronide metabolites. Clin Exp Pharmacol Physiol, 27:524-528, 2000.

[7]. Dwight E. Moulin, Neil G. Johnson, Nancy Murray-Parsons, Margaret F. Geoghegan, Veda A. Goodwin, Margaret A. Chester. Subcutaneous narcotic infusions for cancer pain: treatment outcome and guidelines for use. CMAJ, 146:891-897, 1992.

[8]. David E. Joranson, Karen M. Ryan, Aaron M. Gilson, June L. Dahl. Trends in medical use and abuse of opioid analgesics. JAMA, 283:1710 1714, 2000.

[9]. Laxmaiah Manchikanti, Keith R. Brown, Vijay Singh. National All Schedules Prescription Electronic Reporting Act (NASPER): Balancing Substance Abuse and Medical Necessity. Pain Physician, 5:294-319, 2002.

[10]. Chiara Salvato, Gianfranco Aretini, Donatella Serraglia, Gianni Terrazzani, Patrizia Debetto, Pietro Giusti, Alessandro Chinellato. Opioid prescription for terminally ill outpatients in a district of Northern Italy: a retrospective survey. Pharmacol Res, 48:75-82, 2003.

[11]. Alison Murray and Neil A. Hagen. Hydromorphone. J Pain Symptom Manage, 29:S57-66, 2005.

[12]. Colette M. Reid, Richard M. Martin, Jonathan A. C. Sterne, Andrew N. Davies, Geoffrey W. Hanks. Oxycodone for cancer-related pain: meta analysis of randomized controlled trials. Arch Intern Med, 166:837-843, 2006.

[13]. Peter J. Dunbar, C. Richard Chapman, F. Peter Buckley, Jonathan R. Gavrin. Clinical analgesic equivalence for morphine and hydromorphone with prolonged PCA. Pain, 68:265-270, 1996.

[14]. Martin S. Angst, David R. Drover, John Lotsch, Bhamini Ramaswamy, Sujata Naidu, Russell
Wada, Donald R. Stanski. Pharmacodynamics of orally administered sustained- release hydromorphone in humans. Anesthesiology, 94:63-73, 2001.

[15]. Ellen J. Nickel and Trisha Smith. Analgesia in the intensive care unit. Pharmacologic and pharmacokinetic considerations. Crit Care Nurs Clin North Am, 13:207-219, 2001.

[16]. Julie A. Golembiewski. Morphine and hydromorphone for postoperative analgesia: focus on safety. J Perianesth Nurs, 8:120-122, 2003.

[17]. Julie Golembiewski, Sherri Torrecer, Jennifer Katke. The use of opioids in the postoperative setting: focus on morphine, hydromorphone, and fentanyl. J Perianesth Nurs, 20:141-143, 2005.

[18]. Peter Lawlor, Kenneth Turner, John Hanson, Eduardo Bruera. Dose ratio between morphine and hydromorphone in patients with cancer pain: a retrospective study. Pain, 72:79-85, 1997.

[19]. Barbara Coda, Akira Tanaka, Robert C. Jacobson, Gary Donaldson, C. Richard Chapman. Hydromorphone analgesia after intravenous bolus administration. Pain, 71:41- 48, 1997.

[20]. Joanna L. Hill and James P. Zacny. Comparing the subjective, psychomotor, and physiological effects of intravenous hydromorphone and morphine in healthy volunteers. Psychopharmacology, 152:31-39, 2000.

[21]. Valerie C. Anderson, Beverly Cooke, Kim J. Burchiel. Intrathecal hydromorphone for chronic nonmalignant pain: a retrospective study. Pain Med, 2:287-297, 2001.

[22]. Mary G. Miller, Noel McCarthy, Ciaran A. O'Boyle, Michael Kearney. Continuous subcutaneous infusion of morphine vs. hydromorphone: a controlled trial. J Pain Symptom Manage, 18:9-16, 1999.

[23]. Columba Quigley and Phil Wiffen. A systematic review of hydromorphone in acute and chronic pain. J Pain Symptom Manage, 25:169-178, 2003.

[24]. Paola Sacerdote, Barbara Manfredi, Paolo Mantegazza, Alberto E. Panerai. Antinociceptive and immunosuppressive effects of opiate drugs: a structure-related activity study. $\mathrm{Br}$ J Pharmacol, 121:834-840, 1997.

[25]. J J. Vallner, J T. Stewart, J A. Kotzan, E B. Kirsten, I L. Honigberg. Pharmacokinetics and bioavailability of hydromorphone following intravenous and oral administration to human subjects. J Clin Pharmacol, 21:152-156, 1981.

[26]. Shyi-Feu Chang, Luana Moore, Yie W. Chien. Pharmacokinetics and bioavailability of hydromorphone: effect of various routes of administration. Pharm Res, 5:718-721, 1998. 
[27]. G Pettifer and D Dyson. Hydromorphone: a costeffective alternative to the use of oxymorphone. Can Vet J, 41:135-137, 2000.

[28]. Nabeel Sarhill, Declan Walsh, Kristine A. Nelson. Hydromorphone: pharmacology and clinical applications in cancer patients. Support Care Cancer, 9:84-96, 2001.

[29]. Porter Storey, Herbert H. Hill, Robert H. St Louis, Edward E. Tarver. Subcutaneous infusions for control of cancer symptoms. J Pain Symptom Manage, 5:33-41, 1990.

[30]. B J. Nasits. Dental evaluation of hydromorphone (Dilaudid) for oral and maxillo- facial surgery. Tex Dent J, 87:4-6, 1969.

[31]. Neil A. Hagen and Najib Babul. Comparative clinical efficacy and safety of a novel controlledrelease oxycodone formulation and controlledrelease hydromorphone in the treatment of cancer pain. Cancer, 79:1428-1437, 1997.

[32]. Barbara A. Coda, Barabara O'Sullivan, Gary Donaldson, Sharol Bohl, C. Richard Chapman, Danny D. Shen. Comparative efficacy of patientcontrolled administration of morphine, hydromorphone, or sufentanil for the treatment of oral mucositis pain following bone marrow transplantation. Pain, 72:333-346, 1997.

[33]. John J. Collins, Joanne Geake, Holcombe E. Grier, Constance S. Houuck, Howard T. Thaler, Howard J. Weinstein, Nana Yeboaa Twum- Danso, Charles B. Berde. Patient-controlled analgesia for mucositis pain in children: a three-period crossover study comparing morphine and hydromorphone. J Pediatr, 129:722-728, 1996.

[34]. E V. Deutsch. Postoperative analgesia with hydromorphone and meperidine: a double-blind comparison. Anesth Analg, 47:669-671, 1968.

[35]. Suzanne E. Rapp, Kelly J. Egan, Brian K. Ross, Lorie M. Wild, Gregory W. Terman, Joanie M. Ching. A multidimensional comparison of morphine and hydromorphone patient-controlled analgesia. Anesth Analg, 82:1043- 1048, 1996.

[36]. Stanley L. Wallenstein, Raymond W. Houde, Russell Portenoy, Jeanne Lapin, Ada Rogers, Kathleen M. Foley. Clinical analgesic assay of repeated and single doses of heroin and hydromorphone. Pain, 41:5-13, 1990.

[37]. S R. Chaplan, S R. Duncan, J B. Brodsky, W G. Brose. Morphine and hydromorphone epidural analgesia. A prospective, randomized comparison. Anesthesiology, 77:1090-1094, 1992.

[38]. D H. Chestnut, W W. Choi, T J. Isbell. Epidural hydromorphone for postcesarean analgesia. Obstet Gynecol, 68:65-69, 1986.

[39]. Mashallah Goodarzi. Comparison of epidural morphine, hydromorphone and fentanyl for postoperative pain control in children undergoing orthopaedic surgery. Paediatr Anaesth, 9:419-422, 1999.

[40]. Stephen H. Halpern, Ramiro Arellano, Roanne Preston, Gerry O'Leary, Alan Sandler. Epidural morphine vs hydromorphone in post-caesarean section patients. Can J Anaesth, 43:595-598, 1996.

[41]. Charles E. Inturrisi. Clinical pharmacology of opioids for pain. Clin J Pain, 18:S3-13, 2002.

[42]. Palladone for chronic pain. Med Lett Drugs Ther, 47:21-23,2005.

[43]. Celene M. Amabile and Bill J. Bowman. Overview of oral modified-release opioid products for the management of chronic pain. Ann Pharmacother, 40:1327-1335, 2006.

[44]. FDA asks Purdue Pharma to withdraw Palladone. FDA Consumer, 7, 2005.

[45]. Hydromorphone extended release suspended. WHO Drug Information, 19:217, 2005.

[46]. Nathan J. Cherny, Victor Chang, Gerri Frager, Jane M. Ingham, Paul J. Tiseo, Beth Popp, Russell K. Portenoy, Kathleen M. Foley. Opioid pharmacotherapy in the management of cancer pain: a survey of strategies used by pain physicians for the selection of analgesic drugs and routes of administration. Cancer, 76:1283-1293, 1995.

[47]. Anita C. Rudy, Barbara A. Coda, Sanford M. Archer, Daniel P. Wermeling. A Multiple-Dose Phase I Study of Intranasal Hydromorphone Hydrochloride in Healthy Volunteers. Ambulatory Anesthesia, 99:1379-1386, 2004.

[48]. Eduardo Bruera. Alternate routes for home opioid therapy. Pain: Clinical Updates, 1, 1993.

[49]. Sebastiano Mercadante and Fabio Fulfaro. Alternatives to oral opioids for cancer pain. Oncology, 13:215-225, 1999.

[50]. Robert F. Reder. Opioid formulations: tailoring to the needs in chronic pain. Eur J Pain, 5 Suppl A:109-11, 2005.

[51]. Marcello De Cicco, Roberto Bortolussi, Dario Fantin, Mira Matovic, Augusto Fracasso, Fabio Fabiani, Cristina Santantonio. Supportive therapy of elderly cancer patients. Crit Rev Oncol/Hemat, 42:189-211, 2002.

[52]. K Bloor, B Lesse, A Maynard. The costs of managing severe cancer pain and potential savings from transdermal administration. Eur $\mathrm{J}$ Cancer, 30A:463-468, 1994.

[53]. Wolfgang A. Ritschel, Prakash V. Parab, Donald D. Denson, Dennis E. Coyle, Richard V. Gregg. Absolute bioavailability of hydromorphone after peroral and rectal administration in humans: saliva/plasma ratio and clinical effects. J Clin Pharmacol, 27:647-653, 1987.

[54]. George A. Davis, Anita C. Rudy, Sanford M. Archer, Daniel P. Wermeling, Patrick J. 
McNamara. Effect of fluticasone propionate nasal spray on bioavailability of intranasal hydromorphone hydrochloride in patients with allergic rhinitis. Pharmacotherapy, 24:26-32, 2004.

[55]. Neil Hagen, Michael P. Thirlwell, H S. Dhaliwal, Najib Babul, Zoltan Harsanyi, Andrew C. Darke. Steady-state pharmacokinetics of hydromorphone and hydromorphone-3-glucuronide in cancer patients after immediate and controlled-release hydromorphone. J Clin Pharmacol, 35:37-44, 1995.

[56]. Helen Hays, Neil Hagen, Michael Thirlwell, H. Dhaliwal, Najib Babul, Zoltan Harsanyi, Andrew C. Darke. Comparative clinical efficacy and safety of immediate release and controlled release hydromorphone for chronic severe cancer pain. Cancer, 74:1808-1816, 1994.

[57]. Eduardo Bruera, Jose Pereira, Sharon Watanabe, Michelle Belzile, Norma Kuehn, John Hanson. Opioid rotation in patients with cancer pain. A retrospective comparison of dose ratios between methadone, hydromorphone, and morphine. Cancer, 78:852-857, 1996.

[58]. David R. Drover, Martin S. Angst, Marta Valle, Bhamini Ramaswamy, Sujata Naidu, Donald R. Stanski, Davide Verotta. Input characteristics and bioavailability after administration of immediate and a new extended-release formulation of hydromorphone in healthy volunteers.

Anesthesiology, 97:827-836, 2002.

[59]. Vijay Vashi, Stephen Harris, Ahmed El-Tahtawy, Danlin Wu, Alessandra Cipriano. Clinical pharmacology and pharmacokinetics of oncedaily hydromorphone hydrochloride extendedrelease capsules. J Clin Pharmacol, 45:547-554, 2005.

[60]. Alan B. Grosset, Michael S. Roberts, Mark E. Woodson, Minggao Shi, Ruth E. Swanton, Robert F. Reder, Barbara J. Buckley. Comparative efficacy of oral extended-release hydromorphone and immediate-release hydromorphone in patients with persistent moderate to severe pain: two randomized controlled trials. J Pain Symptom Manage, 29:584-594, 2005.

[61]. Robert Conley, Suneel K. Gupta, Gayatri Sathyan. Clinical spectrum of the osmotic- controlled release oral delivery system (OROS), an advanced oral delivery form. Curr Med Res Opin, 22:1879-1892, 2006.

[62]. Mark Palangio, Donald W. Northfelt, Russell K. Portenoy, Daniel Brookoff, Ralph T. Doyle, Bruce E. Dornseif, Michael C. Damask. Dose conversion and titration with a novel, once-daily, OROS osmotic technology, extended-release hydromorphone formulation in the treatment of chronic malignant or nonmalignant pain. J Pain Symptom Manage, 23:355-368, 2002.

[63]. Suneel K Gupta, Gayatri Sathyan. Providing constant analgesia with OROS $^{\circledR}$ hydromorphone. J Pain Symptom Manage, 33:S19-24, 2007.

[64]. Eduardo Bruera, Paul Sloan, Balfour Mount, Maria Suarez-Almazar. A randomized, doubleblind, double-dummy, crossover trial comparing the safety and efficacy of oral sustained-release hydromorphone with immediate-release hydromorphone in patients with cancer pain. Canadian Palliative Care Clinical Trials Group. J Clin Oncol, 14:1713-1717, 1996.

[65]. K Wegner, S A. Robertson, C Kollias-Baker, R A. Sams, W W. Muir III. Pharmacokinetic and pharmacodynamic evaluation of intravenous hydromorphone in cats. J Vet Pharmacol Therap, 27: 329-336, 2004.

[66]. Eduardo Bruera, C Brenneis, M Michaud, R Bacovsky, S Chadwick, A Emeno, N MacDonald. Use of the subcutaneous route for the administration of narcotics in patients with cancer pain. Cancer, 62:407-441, 1988.

[67]. Russell K. Portenoy, Dwight E. Moulin, Ada Rogers, Charles E. Inturrisi, Kathleen M. Foley. I.V. infusion of opioids for cancer pain: clinical review and guidelines for use. Cancer Treat Rep, 70:575-581, 1986.

[68]. D E. Moulin, J H. Kreeft, N Murray-Parsons N, A I. Bouquillon. Comparison of continuous subcutaneous and intravenous hydromorphone infusions for management of cancer pain. Lancet, 337:465-468, 1991.

[69]. Eduardo Bruera, Carleen Brenneis, Mary Michaud, Sharon Chadwick, R N. MacDoanld. Continuous sc infusion of narcotics using a portable disposable device in patients with advanced cancer. Cancer Treat Rep, 71:635-637, 1987.

[70]. Deborah J. Rhodes and Stuart A. Grossman. Hydromorphone polymer implant. A potential alternative to methadone maintenance. J Subst Abuse Treat, 14:535-542, 1997.

[71]. D Sendil-Keskin, H Altunay, D L. Wise, V Hasirci. In vivo pain relief effectiveness of an analgesic-anesthetic carrying biodegradable controlled release rod systems. J Biomater Sci Polym Ed, 14:497-514, 2003.

[72]. Stuart A. Grossman, Kam W. Leong, Glenn J. Lesser, Hungnan Lo. Subcutaneous implant for delivery of hydromorphone. United States Patent 5858388, 1999.

[73]. D Sendil, I M. Bonney, D B. Carr, L W. Lipkowski, D L. Wise, V Hasirci. Antinociceptive effects of hydromorphone, bupivacaine and biphalin released from PLGA 
polymer after intrathecal implantation in rats. Biomaterials, 24:1969-1976, 2003.

[74]. Roelien H. Enting, Carlo Mucchiano, Wendy Oldenmenger, Magdalena Fritzon, Anna Wallen, Simone Goslinga-van der Gaag, Peter A.E. Sillevis Smitt, Elmar Delhaas. The "pain pen" for breakthrough cancer pain: a promising treatment. J Pain Symptom Manage, 29:213-217, 2005.

[75]. Morphine in cancer pain: modes of administration. BMJ, 312:823-826, 1996.

[76]. J M. Alexander-Williams and D J. Rowbotham. Novel routes of opioid administration. $\mathrm{Br} \mathrm{J}$ Anaesth, 81:3-7, 1998.

[77]. R V. Padmanabhan, J B. Phipps, G A. Lattin, R J. Sawchuk. In vitro and in vivo evaluation of transdermal iontophoretic delivery of hydromorphone. J Contr Rel, 11:123-135, 1990.

[78]. A Smith, E Gomez-Panzani, S Mills, G Rainey, G Tolia, F Tagliaferri, V Spratlin. 24-hour transdermal delivery of hydromorphone hydrochloride. Conference Proceeding, S52, 2007.

[79]. Barbara A. Coda, Anita C. Rudy, Sanford M. Archer, Daniel P. Wermeling. Pharmacokinetics and bioavailability of single-dose intranasal hydromorphone hydrochloride in healthy volunteers. Anesth Analg, 97:117-123, 2003.

[80]. Rhonda R. Pickett, Susan J. Secor. High-tech pain management utilizing multiple analgesics-a case study. Am J Hosp Palliat Care, 12:22-24, 1995.

[81]. Divakara Kedlaya, Lowell Reynolds, Stephen Waldman. Epidural and intrathecal analgesia for cancer pain. Best Pract Res Clin Anaesthesiol, 16:651-665, 2002.
[82]. Mary J. Johansen, William C. Satterfield, Wallace B. Baze, Keith R. Hildebrand, Tamara Lee Gradert, Samuel J. Hassenbusch. Continuous intrathecal infusion of hydromorphone: safety in the sheep model and clinical implications. Pain Med, 5:14-25, 2004.

[83]. Stuart Du Pen, Anna Du Pen, Jon Hillyer. Intrathecal hydromorphone for intractable nonmalignant pain: a retrospective study. Pain Med, 7:10-15, 2006.

[84]. Gary Bennett, Tim Deer, Stuart Du Pen, Richard Rauck, Tony Yaksh, Samuel J. Hassenbusch. Future directions in the management of pain by intraspinal drug delivery. J Pain Symptom Manage, 20:S44-S50, 2000.

[85]. Patrick J. Coyne, Lea Ann Hansen, Jill Laird, Patricia Buster, Thomas J. Smith. Massive hydromorphone dose delivered subcutaneously instead of intrathecally: guidelines for prevention and management of opioid, local anesthetic, and clonidine overdose. J Pain Symptom Manage, 28:273-276, 2004.

[86]. S K. Henderson, E B. Matthew, H Cohen, M J. Avram. Epidural hydromorphone: a double-blind comparison with intramuscular hydromorphone for postcesarean section analgesia. Anesthesiology, 66:825-830, 1987.

[87]. Aaron M. Gilson, Karen M. Ryan, David E. Joranson, June L. Dahl. A reassessment of trends in the medical use and abuse of opioid analgesics and implications for diversion control: 1997-2002. J Pain Symptom Manage, 28:176-188, 2004. 